\title{
Recombination via tail states in polythiophene:fullerene solar cells
}

\author{
Thomas Kirchartz, ${ }^{1}$ Bart E. Pieters, ${ }^{2}$ James Kirkpatrick, ${ }^{3}$ Uwe Rau, ${ }^{2}$ and Jenny Nelson ${ }^{1}$ \\ ${ }^{1}$ Department of Physics, Imperial College London, South Kensington SW7 2AZ, United Kingdom \\ ${ }^{2}$ IEK5-Photovoltaik, Forschungszentrum Jülich, D-52425 Jülich, Germany \\ ${ }^{3}$ Department of Physics, Oxford University, Parks Road, Oxford OX1 3LB, United Kingdom
}

(Received 25 October 2010; revised manuscript received 1 February 2011; published 29 March 2011)

\begin{abstract}
State-of-the-art models used for drift-diffusion simulations of organic bulk heterojunction solar cells based on band transport are not capable of reproducing the voltage dependence of dark current density and carrier concentration of such devices, as determined by current-voltage and charge-extraction measurements. Here, we show how to correctly reproduce this experimental data by including an exponential tail of localized states into the density of states for both electrons and holes, and allowing recombination to occur between free charge carriers and charge carriers trapped in these states. When this recombination via tail states is included, the dependence of charge-carrier concentration on voltage is distinctly different from the case of band-to-band recombination and the dependence of recombination current on carrier concentration to a power higher than 2 can be explained.
\end{abstract}

DOI: 10.1103/PhysRevB.83.115209

\section{INTRODUCTION}

The most promising candidates for future generations of low-cost, thin-film solar cells that can be processed on flexible substrates ${ }^{1-6}$ in roll-to-roll processes are two very different material systems: amorphous and microcrystalline, hydrogenated silicon on the one side and polymer:fullerene $e^{7,8}$ solar cells on the other side. On first sight, the two technologies seem to be quite different, with the traditional semiconductor $\mathrm{Si}$ with decades of research experience on the one side and with the emerging field of polymer optoelectronics on the other side. It has been proposed to distinguish between two distinct types of solar cells, namely, organic or excitonic solar cells and conventional bipolar solar cells, from an inorganic absorber material. ${ }^{9-11}$ In organic solar cells, photon absorption first leads to the generation of excitons, which will then be separated at a heterointerface into free electrons and holes. The relevance of excitons in the process chain between photons and free charge carriers is a fundamental difference between organic materials with their inherently lower dielectric constants than inorganic absorbers. For optimized organic solar cells, however, the exciton diffusion process is no longer a limiting factor. ${ }^{12,13}$ Instead, the shape of the current-voltage curves and the efficiencies seem to depend primarily on nonradiative recombination processes ${ }^{14,15}$ and on the mobilities of the charge carriers just as in the case of inorganic solar cells. These recombination and transport processes are in turn heavily influenced by the intrinsic disorder in the electronic processes within organic semiconductor films. To understand, optimize, and predict the performance of organic bulk-heterojunction solar cells, a suitable theoretical description of the charge transport and recombination processes for use in macroscopic device simulations would be required that takes into account effects of disorder and charge trapping as well as suitable recombination processes and that is capable of reproducing dark and illuminated current-voltage curves. Such a description is currently not available for organic solar cells, but it is well established for disordered inorganic absorber materials as the above-mentioned amorphous and microcrystalline, hydrogenated silicon.

This paper shows that the consideration of trapping and recombination via exponential tails in the valence
PACS number(s): 84.60.Jt, 73.61.Ph, 72.80.Ng, 72.20.Jv

and conduction band of polymer-fullerene solar cells allows us to reproduce experimental data on the currentvoltage curve under illumination and in the dark of a poly (3-hexylthiophene) (P3HT): 1-(3-methoxycarbonyl)propyl-1phenyl-[6,6]-methano fullerene (PCBM) bulk heterojunction solar cell. In addition, we show the consistency of the model with charge-extraction ${ }^{16,17}$ measurements determining the charge-carrier concentration under illumination and in the dark. While the inclusion of charge trapping has been frequently proposed to explain the charge-carrier dependence of mobility ${ }^{18-20}$ and recombination coefficient ${ }^{21,22}$ in organic solar cells, the recombination via tail states has only recently ${ }^{23-27}$ been considered to be of relevance for the description of such devices. Here we show the crucial importance of tail-state recombination for the description of experimental data, especially of the ideality factor under illumination and in the dark.

\section{FUNDAMENTAL THEORY}

For a macroscopic simulation of a semiconductor diode in steady state, three coupled differential equations have to be solved. These equations are the Poisson equation,

$$
\Delta \varphi=-\frac{\rho}{\varepsilon},
$$

relating the electrical potential $\varphi$ to the space charge $\rho$ and the dielectric constant $\varepsilon$, and the drift diffusion equations for the electrons and holes,

$$
\begin{aligned}
-\frac{1}{q} \frac{d J_{\mathrm{n}}(x)}{d x} & =-D_{\mathrm{n}} \frac{d^{2} n(x)}{d x^{2}}-F \mu_{n} \frac{d n(x)}{d x} \\
& =G(x)-R(x, n, p), \\
\frac{1}{q} \frac{d J_{\mathrm{p}}(x)}{d x} & =-D_{\mathrm{p}} \frac{d^{2} p(x)}{d x^{2}}+F \mu_{p} \frac{d p(x)}{d x} \\
& =G(x)-R(x, n, p),
\end{aligned}
$$

where $x$ is the spatial coordinate normal to the cell surface, $n$ and $\mathrm{p}$ are the concentrations of free electrons and holes, respectively, $J_{n}$ and $J_{p}$ are the electrical electron and the hole current densities, and $G$ and $R$ are the generation and 
recombination rates of free charge carriers. The diffusion constants $D_{\mathrm{n}, \mathrm{p}}$ are assumed to be connected via the simple Einstein relation $\left(D_{\mathrm{n}, \mathrm{p}}=\mu_{\mathrm{n}, \mathrm{p}} k T / q\right)$ to the electron and hole mobilities $\mu_{n}$ and $\mu$ and the thermal voltage $k T / q$. We will later discuss the implications of deviations from the Einstein relation.

The boundary condition for the electrical potential is

$$
\varphi(d)-\varphi(0)=V_{\mathrm{bi}}-V,
$$

defining the potential difference between the potential $\varphi(d)$ at the back contact and $\varphi(0)$ at the front contact as a function of the built-in voltage $V_{\mathrm{bi}}$ and the applied voltage $V$. The boundary conditions for the currents and the carrier concentrations at both contacts are

$$
\begin{aligned}
& J_{\mathrm{n}}(0)=q S_{\mathrm{nf}}\left[n(0)-n_{0}(0)\right], \\
& J_{\mathrm{p}}(0)=q S_{\mathrm{pf}}\left[p(0)-p_{0}(0)\right], \\
& J_{\mathrm{n}}(d)=q S_{\mathrm{nb}}\left[n(d)-n_{0}(d)\right], \\
& J_{\mathrm{p}}(d)=q S_{\mathrm{pb}}\left[p(d)-p_{0}(d)\right],
\end{aligned}
$$

where $S_{\mathrm{nf}, \mathrm{b}}$ is the surface recombination velocity for electrons at the front or back, respectively, while $S_{\mathrm{pf}, \mathrm{b}}$ is the analogous quantity for the holes. Equations (1)-(5) allow us to calculate the local carrier concentrations and the electrostatic potential as a function of applied voltage $V$ and incident photon flux. From the carrier concentrations, the currents follow either by using Eq. (5) or by integrating Eqs. (2) and (3), such that a complete current-voltage curve under illumination or in the dark is simulated.

Up to now, we reviewed the features of most of the available drift-diffusion simulators that are commonly used for solar-cell modeling in general. Effective-medium models based on the theory outlined above have been routinely used to model not only inorganic thin-film solar cells but also organic bulk heterojunction solar cells. In the case of the bulk-heterojunction solar cell, the lowest unoccupied molecular orbital (LUMO) of the acceptor and the highest occupied molecular orbital (HOMO) of the donor are considered in an analogous way to the conduction and valence band in an inorganic solar cell. Despite the fact that the bulk heterojunction architecture consists of a blend of two different materials, drift-diffusion-based effective-medium approaches are still very useful to interpret device measurements, as has been shown numerous times. ${ }^{28-35}$

The basic differences between the models typically used for disordered inorganic solar cells, on the one hand, and organic solar cells, on the other hand, are the choice of generation rate $G$ and recombination rate $R$. While for inorganic solar cells, the generation rate simply follows from the optical properties of the device such as absorption coefficient and thickness, the generation rate for free carriers in organic solar cells is often assumed to be field dependent, according to different models as described in Refs. 36-40. This electric-field-dependent generation rate is rationalized with the field-dependent dissociation of the charge-transfer (CT) state exciton at the heterointerface between a donor and acceptor molecule in a bulk heterojunction solar cell. However, recent experimental data shows that for typical organic bulk heterojunction solar cells based on P3HT:PCBM, the field dependence of free carrier generation cannot have a major effect on the current-voltage curve, ${ }^{41-43}$ while Monte Carlo simulations showed that in good cells the field dependence of geminate recombination is small in the relevant range of electric fields. ${ }^{44}$ Thus, in our simulations we neglect the field dependence of the generation rate and use a spatially independent rate.

Despite the complexity of the generation rate usually assumed in device simulations of organic solar cells, the recombination rate, however, is mostly assumed to follow very simple equations, namely, direct electron-hole recombination with a prefactor given by the Langevin theory. These assumptions lead to a recombination rate given by ${ }^{39}$

$$
R=\frac{q\left(\mu_{\mathrm{n}}+\mu_{\mathrm{p}}\right)}{\varepsilon}\left(n p-n_{\mathrm{i}}^{2}\right),
$$

where $n_{i}$ is the intrinsic carrier concentration. The form of the recombination rate in Eq. (6) ensures that there is no recombination when there are no (optically or electrically created) excess carriers available, i.e., when $n p=n_{\mathrm{i}}^{2}$. The field-dependent generation rate combined with the simple Langevin recombination rate was sufficient to reproduce the experimental results for the current-voltage $(J-V)$ curve under illumination. However, the $J-V$ curve in the dark cannot be reproduced with these assumptions. The dark current density $J_{\mathrm{d}}$ is a direct measure of the spatially integrated recombination rate, and it can be written as

$$
J_{\mathrm{d}}=q \int_{0}^{d} R(x) d x+q S_{\mathrm{n}} n(0)+q S_{\mathrm{p}} p(d)
$$

for the case when the electron contact is at $x=d$ and the hole contact is at $x=0$. The dark ideality factor,

$$
n_{\mathrm{id}}=\frac{q}{k T} \frac{d V}{d \ln \left(J_{d}\right)},
$$

follows from the slope of the dark $J-V$ curves in the voltage range where they are neither dominated by shunt or series resistances. Typical values are in the range $1<n_{\text {id }}<2$. However, the values for $n_{\text {id }}$ are typically considerably larger than 1 , meaning that they cannot be explained anymore by simple direct recombination as assumed in Eq. (6), which would lead to $n_{\text {id }}=1$. Recently, various measurements of the carrier-concentration-dependent recombination rate ${ }^{16,45-50}$ have suggested an empirical form of the recombination rate according to

$$
R=k(n) n p=k_{0} n^{\lambda} n p,
$$

with $\lambda>0$. Such a recombination rate resembles Auger recombination, which leads to dark ideality factors $n_{\mathrm{id}}<1$ (see Appendix A).

The failure of all recombination models typically used for the simulation of organic solar cells to reproduce the observed dark ideality factors motivates the search for more appropriate models. One possible source for such models is the literature $^{51}$ on disordered inorganic solar cells. As shown in Fig. 1, the above-mentioned amorphous and microcrystalline silicon solar cells have similar $J-V$ curves in the dark and similar dark ideality factors as a typical organic solar cell with a P3HT:PCBM absorber layer. The reason why models developed for thin-film silicon solar cells are capable of reproducing the experimental dark ideality factors is the inclusion of localized subband-gap states that are due to defects or disorder 


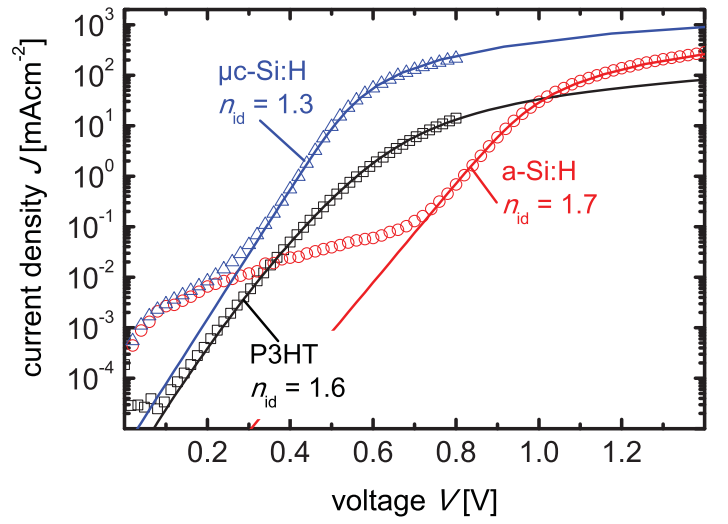

FIG. 1. (Color online) Comparison of experimental dark currentvoltage curves (symbols) of an organic solar cell based on a P3HT:PCBM absorber with two representative thin-film silicon solar cells. All three cells show a clear diode behavior with noninteger dark ideality factors. The solid lines for the two silicon solar cells are one-diode fits to determine the ideality factor, and the solid line in the case of the P3HT:PCBM cell is a simulation using the parameters detailed in Table I, column 2.

and that are actively involved in recombination..$^{52-54}$ Thus, even in completely defect-free organic solar cells, the inherent disorder in molecular packing and molecular environment should lead to subband-gap states that could contribute to charge-carrier recombination. In the following, we will show how inclusion of exponential tail states occupied by nonmobile or trapped charge carriers and recombination via these states allows us to reproduce experimental data that cannot be reproduced by only assuming free carriers in bands.

In addition to an exponentially decaying tail, in thin-film silicon solar cells it is common to include a distribution of deep trap states and to allow recombination between free carriers and such deep states. This is also a possible mechanism for organic solar cells, as suggested, e.g., by Street et al. ${ }^{55}$ However, it is not taken into account here, since it increases the number of parameters, and since it can be shown easily that any data that can be modeled by including both tail recombination and recombination via deep states can also be reproduced by a model including tail recombination only.

Figure 2 presents an overview over the effect of tail states on transport and recombination. There are four processes that take place. For the valence-band tail states, we have (i) the capture of an electron by a trapped hole (or a positively charged valence-band tail state) with a capture rate coefficient given by $\beta_{\mathrm{n}}^{+}\left([\beta]=\mathrm{cm}^{3} / \mathrm{s}\right)$, (ii) the capture of a hole by an unoccupied (i.e., neutral) valence-band state with a capture rate coefficient given by $\beta_{\mathrm{p}}^{0}$, (iii) the capture of a hole by a negatively charged conduction-band state with a capture rate coefficient given by $\beta_{\mathrm{p}}^{-}$, and (iv) the capture of an electron by an unoccupied (i.e., neutral) conduction-band state with a capture rate coefficient given by $\beta_{\mathrm{n}}^{0}$.

The idea behind the tail recombination model is that the recombination of free carriers via tails is modeled with an effective recombination rate that takes the rate equations for recombination, trapping, and detrapping into account and calculates the net recombination rate. The transport model is a multiple trapping model, i.e., transport is affected by the

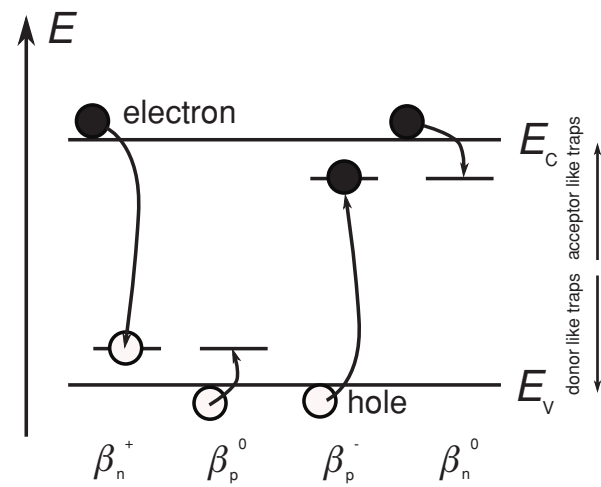

FIG. 2. Schematics visualizing the definition of the four capture rate coefficients for capture and recombination via tail states. From left to right, the capture rate coefficients describe (i) recombination of an electron with a trapped hole in the valence-band tail, (ii) trapping of a hole by a neutral valence-band tail state, (iii) recombination of a free hole with a trapped electron in a conduction-band tail state, and (iv) trapping of an electron by a neutral conduction-band tail state. Note that the unit of all capture rate coefficients is $\mathrm{cm}^{3} \mathrm{~s}^{-1}$.

trapping and detrapping processes. The effect of the multiple trapping model on the effective mobility is that it becomes implicitly carrier concentration dependent since only a small part of the total charge carriers contribute to the transport. The terminology of free carriers is traditionally used in disordered inorganic solar cells, however, it might be misleading in the case of the transport via localized states in organic semiconductors. Here, free means that these carriers are sufficiently far away from midgap that the Boltzmann approximation can be used to calculate their concentration for the whole range of voltages studied ( $V<V_{\mathrm{oc}}$ at 1 sun) and that their mobility is nonzero. All other states are tail states, which are assumed to be completely localized and whose occupation probability has to be calculated with the Shockley-Read-Hall (SRH) formalism and not with Boltzmann approximation. The approximation involved is that the transition between free and trapped carriers is abrupt. Reference 56 shows that this approximation only affects the temperature dependence of the current-voltage curve, while it has only the effect of a constant scaling factor for the mobility when temperature is kept constant.

The recombination rate used to simulate semiconductors with exponential tail states follows from SRH statistics ${ }^{57,58}$ as a function of the electron and hole capture rate coefficients $\beta_{\mathrm{n}, \mathrm{p}}$, of the energy-dependent density $N_{\mathrm{CBT}} / N_{\mathrm{VBT}}$, of conductionvalence band states, given by the exponential decay of the tail defined by the Urbach energy $E_{\mathrm{UC}}$ and $E_{\mathrm{UV}}$ for both tails. Note that SRH statistics imply that recombination requires at least one free carrier, i.e., there will be no recombination between trapped electrons and trapped holes. Instead, one recombination process consists of trapping of a free electron and recombination of this trapped electron with a free hole or vice versa of a trapped hole with a free electron. The recombination rate is expressed as an integral from valence-band edge $E_{\mathrm{V}}$ to the conduction-band edge $E_{\mathrm{C}}$ over the density of tail states and the recombination efficiency $\eta_{\mathrm{R}}$, which follows from the detailed balance between capture and emission processes. The complete equation for the recombination rate $R_{\mathrm{CBT}}$ via 
conduction-band states is given by ${ }^{59}$

$$
\begin{aligned}
R_{\mathrm{CBT}} & =\int_{E_{\mathrm{V}}}^{E_{\mathrm{C}}} N_{\mathrm{CBT}}(E) \eta_{\mathrm{R}}(E) d E \\
& =\int_{E_{\mathrm{V}}}^{E_{\mathrm{C}}} N_{\mathrm{C} 0} \exp \left(\frac{E-E_{\mathrm{C}}}{E_{\mathrm{UC}}}\right) \frac{n p-n_{i}^{2}}{\left(n+N_{\mathrm{C}} \exp \left[\left(E-E_{\mathrm{C}}\right) / k T\right]\right) / \beta_{\mathrm{p}}^{-}+\left(p+N_{\mathrm{V}} \exp \left[\left(E_{\mathrm{V}}-E\right) / k T\right]\right) / \beta_{\mathrm{n}}^{0}} d E,
\end{aligned}
$$

where $N_{\mathrm{C} 0}$ is the density of tail states per energy interval at the conduction-band edge. The total recombination rate to be inserted in Eqs. (2) and (3) is then $R=R_{\mathrm{CBT}}+R_{\mathrm{VBT}}$, where the recombination rate $R_{\mathrm{VBT}}$ via valence-band tail states follows from a similar equation as given by Eq. (10). Note that the capture rate coefficients $\beta_{\mathrm{p}}^{-}, \beta_{\mathrm{n}}^{0}$ for the conduction-band tail and $\beta_{\mathrm{p}}^{0}, \beta_{\mathrm{n}}^{+}$for the valence-band tail are often expressed as the product of thermal velocity $v_{\text {th }}$ and capture cross sections $\sigma_{\mathrm{n}, \mathrm{p}}$. However, to consider thermal velocity $v_{\text {th }}$ and capture cross section as individual parameters does not have any benefit ${ }^{60}$ for the description of thin-film solar cells. Thus, we only use capture rate coefficients in the following, which have the unit $\mathrm{cm}^{3} \mathrm{~s}^{-1}$

Note that the recombination via these tail states is expected to be predominantly nonradiative, as indicated by the low luminescence yield measured by Vandewal et al. ${ }^{14,61}$ However, the measured light emission may very well originate from recombination via tail states, which has implications for the modeling and interpretation of luminescence as shown for the case of microcrystalline silicon. ${ }^{62}$

Recombination rates as given by Eq. (10) are known to reproduce typical dark ideality factors $1<n_{\text {id }}<2$, as observed in both organic and inorganic $p-i-n$ type solar cells. Thus, in contrast to the commonly used Langevin recombination according to Eq. (6), the mathematical form of Eq. (10) has the potential to describe dark $J-V$ curves of organic solar cells. However, it is not necessary to completely abandon the picture of diffusion-limited recombination. Instead, the capture rates that involve the capture of a hole by an electron trapped in a conduction-band tail state or the capture of an electron by a hole trapped in a valence-band tail state are expected to follow a similar relation as the recombination between a free electron and a free hole. Thus, the Langevin theory would predict the capture rate coefficients,

$$
\beta_{n}^{+}=\frac{q \mu_{\mathrm{n}}}{\varepsilon}
$$

for the capture of a free electron by a hole trapped in a valenceband tail state and

$$
\beta_{\mathrm{p}}^{-}=\frac{q \mu_{\mathrm{p}}}{\varepsilon},
$$

for the capture of a free hole by an electron trapped in a conduction-band tail state.

\section{TAIL-STATE RECOMBINATION VERSUS RECOMBINATION OF FREE CARRIERS}

While the influence of disorder on transport in organic semiconductors is well known, the influence of disorder on recombination is less frequently discussed. Thus, we want to show in the following how the dark ideality factor changes when we go from a situation dominated by recombination between carriers far away from the quasi-Fermi levels to a situation dominated by recombination via tail states. To visualize the effect of the capture rates on the voltage-dependent recombination in organic solar cells, we make simulations using the model described above and the parameters as given in the first column of Table I. We vary the two capture rate coefficients $\beta_{n}^{+}$and $\beta_{p}^{-}$which are responsible for recombination and keep the value of the capture cross sections for carrier trapping constant. In addition to tail-state recombination, we also allow for direct recombination between free carriers with a recombination rate $R=k\left(n p-n_{\mathrm{i}}^{2}\right)$. We simulate both dark and illuminated current-voltage curves and determine the dark ideality factor from the slope of the dark $J-V$ curve using Eq.(8) at every voltage. We then average the dark ideality factors for voltages $0.1 \mathrm{~V}<V<0.6 \mathrm{~V}$ and call the resulting value the apparent dark ideality factor.

Figure 3 shows the open-circuit voltage $V_{\mathrm{oc}}$ and the apparent dark ideality factor resulting from these simulations. The open-circuit voltage for low capture rates is limited by direct recombination and approaches an arbitrary value of $V_{\text {oc }}$ that is given by the chosen generation rate and recombination coefficient for direct recombination $\left(V_{\mathrm{oc}}=857 \mathrm{mV}\right)$. For higher capture rates, the tail-state recombination becomes dominant and decreases $V_{\mathrm{oc}}$.

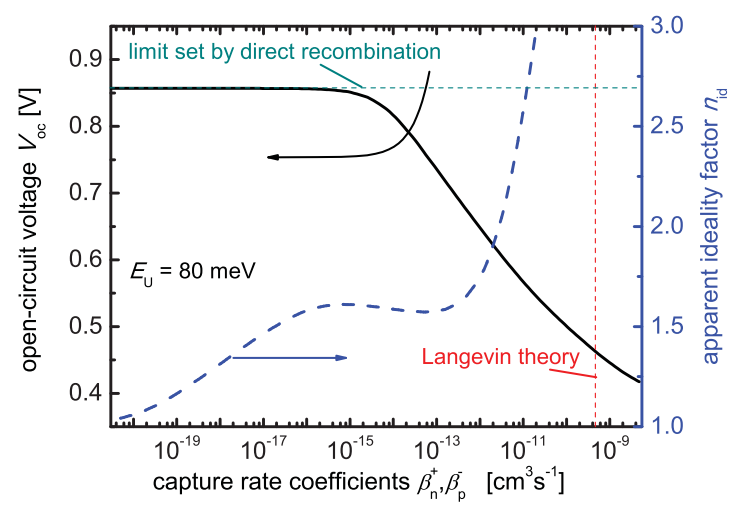

FIG. 3. (Color online) Simulated open-circuit voltage and dark ideality factor of a cell with tails (tail slope $E_{\mathrm{U}}=80 \mathrm{meV}$ for both tails) to show the influence of the two capture rate coefficients for recombination. If these rates are low, there is only trapping but no recombination via the tail states. It is obvious that in this case the dark ideality factor approaches 1 again, since in this case direct recombination would take over. For higher capture rate coefficients, open-circuit voltage decreases and ideality factor increases up to $n_{\text {id }}=1.6$. For higher capture rate coefficients, the true ideality factor is difficult to determine since the series resistance becomes dominant at higher forward voltages. 
TABLE I. Parameters used for the simulations, presented in Fig. 3 (first column) and in Figs. 4, 6, and 7. Note that the mobilities given are band mobilities and apply only to the untrapped fraction of the charge-carrier population. The value of the drift mobility that applies to the general charge-carrier population is substantially smaller than the band mobility, when a multiple trapping model is used. A multiple trapping model is used, when the capture rate coefficients $\beta$, the Urbach energies $E_{\mathrm{UC}, \mathrm{V}}$, and the densities $N_{\mathrm{C} 0}, N_{\mathrm{V} 0}$ of tail states per energy interval at the mobility edge are all nonzero, as in the second column.

\begin{tabular}{|c|c|c|c|c|}
\hline & Cell in Fig. 3 & Cell with tail rec. & Cell with dir. rec. & Cell with Auger rec. \\
\hline$\mu_{\mathrm{n}}\left(\mathrm{cm}^{2} / \mathrm{V} \mathrm{s}\right)$ & $10^{-3}$ & $7.3 \times 10^{-4}$ & $1.7 \times 10^{-4}$ & $8 \times 10^{-5}$ \\
\hline$\mu_{\mathrm{p}}\left(\mathrm{cm}^{2} / \mathrm{V} \mathrm{s}\right)$ & $10^{-3}$ & $7.1 \times 10^{-4}$ & $3 \times 10^{-5}$ & $1.6 \times 10^{-5}$ \\
\hline$N_{\mathrm{C}}\left(\mathrm{cm}^{-3}\right)$ & $10^{20}$ & $10^{20}$ & $10^{21}$ & $10^{21}$ \\
\hline$N_{\mathrm{V}}\left(\mathrm{cm}^{-3}\right)$ & $10^{20}$ & $10^{20}$ & $10^{21}$ & $10^{21}$ \\
\hline$N_{\mathrm{C} 0}\left(\mathrm{~cm}^{-3} \mathrm{eV}^{-1}\right)$ & $10^{20}$ & $3.5 \times 10^{20}$ & 0 & 0 \\
\hline$N_{\mathrm{V} 0}\left(\mathrm{~cm}^{-3} \mathrm{eV}^{-1}\right)$ & $10^{20}$ & $4.7 \times 10^{18}$ & 0 & 0 \\
\hline$E_{\mathrm{UC}}(\mathrm{meV})$ & 80 & 47 & 0 & 0 \\
\hline$E_{\mathrm{UV}}(\mathrm{meV})$ & 80 & 115 & 0 & 0 \\
\hline$\beta_{\mathrm{n}}^{+}\left(\mathrm{cm}^{3} \mathrm{~s}^{-1}\right)$ & Variable & $1.1 \times 10^{-11}$ & 0 & 0 \\
\hline$\beta_{\mathrm{p}}^{0}\left(\mathrm{~cm}^{3} \mathrm{~s}^{-1}\right)$ & $10^{-10}$ & $2.3 \times 10^{-10}$ & 0 & 0 \\
\hline$\beta_{\mathrm{p}}^{-}\left(\mathrm{cm}^{3} \mathrm{~s}^{-1}\right)$ & Variable & $5.2 \times 10^{-13}$ & 0 & 0 \\
\hline$\beta_{\mathrm{n}}^{0}\left(\mathrm{~cm}^{3} \mathrm{~s}^{-1}\right)$ & $10^{-10}$ & $2.6 \times 10^{-10}$ & 0 & 0 \\
\hline$E_{\mathrm{g}}(\mathrm{eV})$ & 1.1 & 1.1 & 1.1 & 1.1 \\
\hline$d(\mathrm{~nm})$ & 150 & 150 & 150 & 150 \\
\hline$k\left(\mathrm{~cm}^{3} \mathrm{~s}^{-1}\right)$ & $2.2 \times 10^{-14}$ & 0 & $7.7 \times 10^{-13}$ & 0 \\
\hline$C_{\text {auger }}\left(\mathrm{cm}^{6} \mathrm{~s}^{-1}\right)$ & 0 & 0 & 0 & $2.7 \times 10^{-30}$ \\
\hline$S(\mathrm{~cm} / \mathrm{s})$ & 0 & $10^{5}$ & $10^{5}$ & $10^{5}$ \\
\hline$\varphi_{\mathrm{b}}(\mathrm{meV})$ & 0 & 0 & 0 & 0 \\
\hline$G\left(\mathrm{~cm}^{-3} \mathrm{~s}^{-1}\right)$ & $1.8 \times 10^{22}$ & $3.85 \times 10^{21}$ & $3.85 \times 10^{21}$ & $3.85 \times 10^{21}$ \\
\hline$\varepsilon_{\mathrm{r}}$ & 3.8 & 3.8 & 3.8 & 3.8 \\
\hline$R_{\mathrm{s}}\left(\Omega \mathrm{cm}^{2}\right)$ & 0 & 6.8 & 5.8 & 4 \\
\hline
\end{tabular}

The apparent dark ideality factor approaches unity for low capture rates as expected for a solar cell where recombination is limited by direct recombination. The dark ideality factor increases to a value $\sim 1.6$, which is the true dark ideality factor (not influenced by series resistances) for tail-state recombination with the assumed Urbach energy $E_{\mathrm{U}}=80 \mathrm{meV}$. For even higher capture rates, the charge transport is hindered and the finite resistance of the absorber layer leads to a voltage drop already at voltages below 0.6 V. Thus, the apparent dark ideality factor is now influenced by the series resistance from the absorber layer itself and starts to increase above 2. This value, however, is no longer an indication of the recombination mechanism. Note that the dark ideality factor rises above 1 for much lower capture rates than the $V_{\text {oc }}$ decreases below the limit given by direct recombination. This is due to the fact that the recombination mechanism with the higher dark ideality factor (i.e., tail-state recombination) becomes relevant at lower bias voltages first and affects the open-circuit voltage only for even higher capture rates.

In this example, using the Langevin theory to determine the capture rates leads to a low open-circuit voltage $V_{\mathrm{oc}}=460 \mathrm{mV}$ as compared to the band-gap energy $E_{\mathrm{g}}=1.1 \mathrm{eV}$, which is an indication that the Langevin theory is indeed too pessimistic, also for the definitions given by Eqs. (11) and (12).

\section{SIMULATION OF CURRENT-VOLTAGE CURVES}

It is well known from experimental and theoretical work on thin-film silicon diodes that $\mathrm{SRH}$ recombination via tail states produces noninteger dark ideality factors in the observed range $1<n_{\mathrm{id}}<2$. $^{63,64}$ Thus, given the relatively large amount of unknown parameters the tail-recombination model contains, it is hardly surprising that it is possible to fit experimental dark $J-V$ curves of a P3HT:PCBM bulk heterojunction device. ${ }^{65}$

A more meaningful test of the potential appropriateness of the model is to compare dark and illuminated $J-V$ curve as well as the illumination-dependent open-circuit voltage in experiment and simulation using always the same set of parameters. Thus, we simulated these three characteristics using the software $\mathrm{ASA}^{66}$ and three different models for recombination, namely, the model with tails, a model with direct recombination, and a model with Auger recombination. ASA was chosen for this purpose due to its numerical robustness and speed and because it is easily controlled by external software. In this case the parameter optimization was done with MATLAB using a downhill simplex algorithm ${ }^{67,68}$ that calls ASA with a different set of parameters in each loop of the algorithm. The algorithm simultaneously minimizes the error in light and dark $J-V$ curves as well as the dark charge-extraction measurements, which will be discussed in Sec. V. The parameters determined in this way are detailed in Table I and are the same for all Figs. 4(a)-4(c). Obviously the parameters should all be determined experimentally and not by fitting to $J-V$ curves. However, our intention is not to determine an adequate parameter set for a certain type of solar cell but to test the consistency of the discussed models with experimental data.

Figure 4(a) shows the experimentally determined dark current-voltage curve (open squares) and the fit by the three models. As expected, models based on Auger recombination 

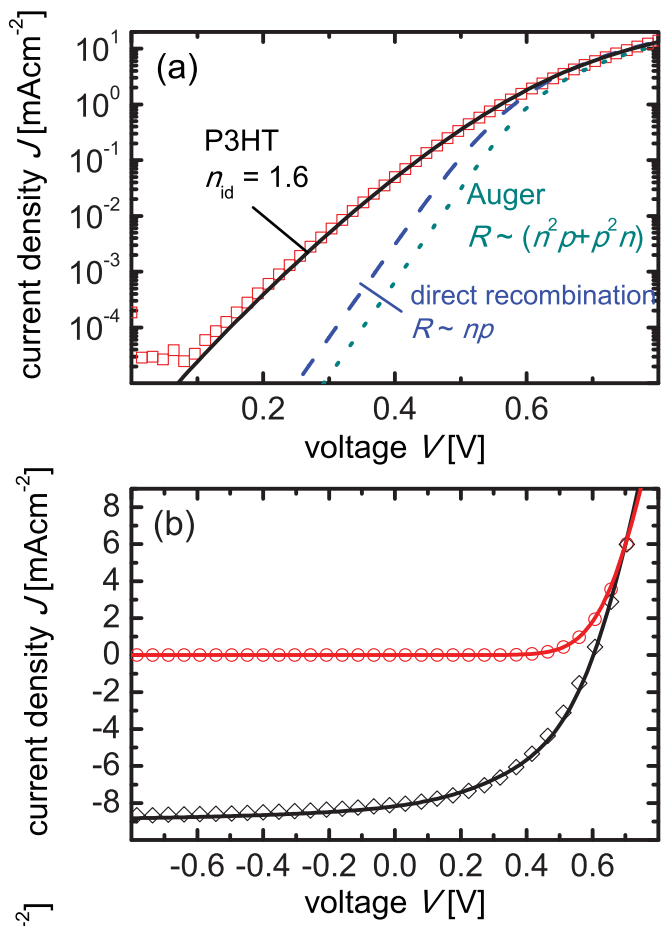

Figure 4(c) shows the illumination-dependent open-circuit voltage from experiment and as simulated by all three models. As a measure of the illumination intensity, the current density at $V=-2 \mathrm{~V}$ is plotted on the ordinate. Such a depiction allows determining the light ideality factor $n_{\mathrm{id}, 1}$, which we define here in analogy to Eq. (8) by

$$
n_{\mathrm{id}, 1}=\frac{q}{k T} \frac{d V_{\mathrm{oc}}}{d \ln [J(-2 V)]} .
$$

The result for the light ideality factor and in the dark is practically identical. The experimental light ideality factor $n_{\text {id }} \approx 1.6$ is well reproduced by the simulation with $\mathrm{SRH}$ recombination via tail states, while direct or Auger recombination models cannot produce light or dark ideality factors $n_{\text {id }}>1$ (see Appendix A).

\section{ANALYSIS OF CHARGE-EXTRACTION MEASUREMENTS}

During dark charge-extraction measurements a voltage is applied to a solar cell before the device is short circuited and the current transient is measured. ${ }^{16}$ The integral of the current transient is then the extracted charge, which consists of all trapped and free excess charge carriers that can be extracted before they would recombine. Charge extraction (CE) can also be measured under illumination. In this case, the device is held at a certain voltage, e.g., the open-circuit voltage, and the light is switched off at the same time as the device is short circuited.

In Ref. 16, CE measurements under illumination and in the dark are presented. The cell used for these measurements was the same as the one used for Fig. 4. The first main conclusion of Ref. 16 was that, both in the dark and under illumination, the current density scales with

$$
J \propto n_{\mathrm{av}}^{\delta},
$$

with $\delta \approx 2.6$, where $n_{\text {av }}$ is the average carrier concentration obtained from the $\mathrm{CE}$ experiment. In addition, the experiments showed a dependence of carrier concentration versus voltage that followed the relation

$$
n_{\mathrm{av}}=n_{0} \exp (\gamma V)
$$

The simulations are preformed using three models with different assumptions for the recombination mechanism (tails, direct, Auger). Only recombination via tail states explains the two semilogarithmic plots (a), (c) from which the dark and light ideality factors follow. The light $J-V$ curve at one intensity (b) is well reproduced by all models, so only the tail recombination model is shown to improve clarity. Parameters for the simulations are detailed in Table I.

or direct recombination are not capable of reproducing dark ideality factors $n_{\text {id }}>1$ and fail to reproduce the experimental data for low voltages, where the diode behavior is visible. For higher voltages, where the curves are dominated by the series resistance, all models fit the experiment well.

Figure 4(b) shows the experimental dark and illuminated $J-V$ curve (open symbols) on a linear scale. The solid lines represent the fit of the tail-recombination model only. Such a good fit to the illuminated $J-V$ curve is reproduced similarly well by all three models, and thus only the fit from one model is shown.

with $\gamma=9.3 \mathrm{~V}^{-1}$ under illumination and $\gamma=7.4 \mathrm{~V}^{-1}$ in the dark. Of course, for self-consistency, the factors $\delta$ and $\gamma$ must be consistent with the observed light or dark ideality factor $n_{\mathrm{id}}$. So for voltages where the $J-V$ curve shows a diode behavior and is not limited by series resistance effects,

$$
\frac{d \ln (J)}{d \ln \left(n_{\mathrm{av}}\right)} \frac{d \ln \left(n_{\mathrm{av}}\right)}{d V}=\delta \gamma=\frac{q}{n_{\mathrm{id}} k T}
$$

must hold. Note that current density $J$ and voltage $V$ in Eq. (16) can be interpreted either as dark current density and applied voltage or as the saturated current density under illumination and reverse bias and the corresponding open-circuit voltage. For the value $\gamma=9.3 \mathrm{~V}^{-1}$ under illumination, $\delta \approx 2.6$, and $k T / q=25.8 \mathrm{mV}$, the resulting light ideality factor is $n_{\mathrm{id}, 1}=$ 1.6, showing the self-consistency of this data. Any reasonable macroscopic model describing the device physics should be able to explain all three slopes, $n_{\mathrm{id}}, \gamma$, and $\delta$.

On first sight, the values $n_{\mathrm{id}}>1$ and $\delta>2$ seem to be inconsistent (cf. Appendix C). While $\delta>2$ suggests, 
for example, a recombination mechanism with a functional dependence of recombination rate on carrier concentration similar to Auger recombination, Auger recombination or also direct recombination between free carriers is inconsistent with the observed dark and light ideality factors. If both $n_{\mathrm{id}}$ and $\delta$ seem to be inconsistent, it is advisable to have a closer look at the remaining factor $\gamma$, which defines the product of $n_{\text {id }}$ and $\delta$. The dependence of carrier concentration and voltage must therefore deviate from our expectations. In a $p-i$ - $n$ device with a built-in field, the general dependence is difficult to obtain except by numerical simulations, since the carrier concentration is strongly position dependent. The electron concentration, e.g., is high at the electron extracting contact and decreases strongly toward the opposite contact due to the built-in electric field in the device. However, this is only true for low voltages and not for open-circuit conditions at reasonably high light intensities. In this case, the built-in voltage is low and the voltage drops mainly in the region close to the contacts, where the space charge, i.e., $q|n-p|$ is large. However, in the largest part of the absorber, $n \approx p$ and the carrier concentrations will only weakly depend on position. Thus, our first expectation would be that both electron and hole concentrations are proportional to $\exp (q V / 2 k T)$. At room temperature, this leads to a slope $\gamma=19.3 \mathrm{~V}^{-1}$, which is a factor of 2 higher than observed.

To understand why the slope $\gamma$ is reduced compared to expectations, we carried out simulations of the average excess carrier concentration as a function of open-circuit voltage for a model with direct recombination and for a model with tail recombination and trapping in tails. The parameters are the same as used for Fig. 3 (first column in Table I), except that the capture rate coefficients for recombination, which were varied for Fig. 3, are kept constant at $\beta=10^{11} \mathrm{~cm}^{3} / \mathrm{s}$ for both carrier types and that the generation rate is varied. The average excess carrier concentrations are calculated such that the equilibrium carrier concentration is subtracted from the carrier concentration under illumination. In case of the model with trapping in tail states, the trapped carriers are included in this calculation. Thus, we assume here that most excess carriers from tail states are extracted faster than they recombine.

Figure 5(a) shows the average carrier concentration as a function of open-circuit voltage for both models in a semilogarithmic plot. The main difference between both models is that the model with tails seems to have a relatively constant slope, while the slope for the model with direct recombination is increasing toward higher open-circuit voltages. Figure 5(b) shows the slope $\gamma$, revealing that the direct recombination model indeed leads to a monotonously increasing slope that converges at $\gamma=1 / 2 k T \approx 19.3 \mathrm{~V}^{-1}$, while the tail recombination model leads to a relatively constant slope with $6 \mathrm{~V}^{-1}<\gamma<8 \mathrm{~V}^{-1}$. The constant increase of the direct recombination model follows from the position dependence of the carrier concentration, which is more pronounced for the case without trapping. The additional trapped charge allows the bands to bend more strongly at the periphery of the absorber so that the carrier concentrations are more homogenous throughout the absorber also for lower voltages. More importantly, however, the trapped charge has a different dependence of carrier concentration on voltage, which mostly depends on the value of the tail slopes. For

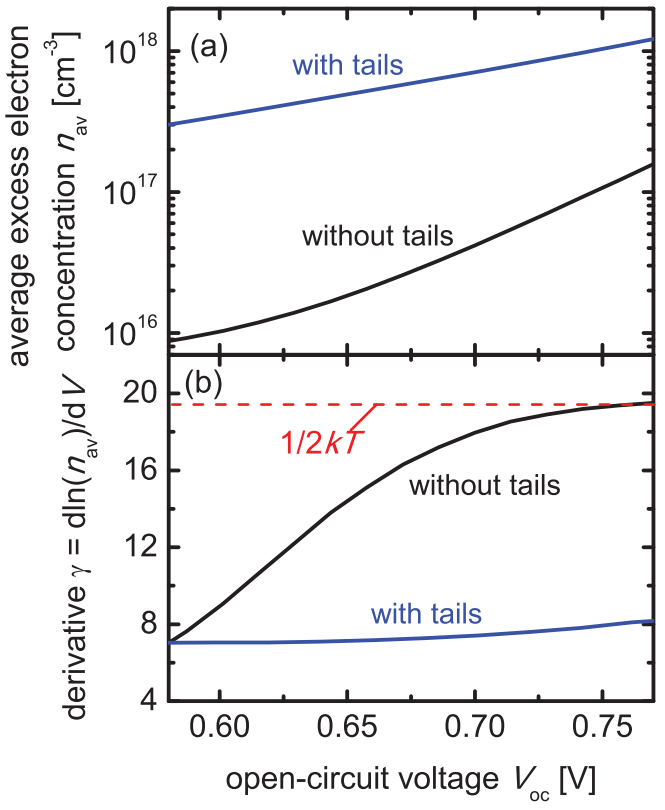

FIG. 5. (Color online) (a) Simulated average carrier concentration (free and trapped) as a function of voltage for a simulation with and without tail states and (b) the corresponding derivatives $\gamma=$ $d \ln \left(n_{\mathrm{av}}\right) / d V_{\mathrm{oc}}$. Without tails, the slope $\gamma$ saturates at $\gamma=1 /(2 k T)$, as expected for the case $n \approx p$ and both having a weak position dependence. Due to the different voltage dependence of the trapped charge, the slope is relatively constant for the simulation with tails and considerably lower than without tails.

high Urbach energies $E_{\mathrm{U}} \gg k T$, the increase of the sum of trapped and free carrier concentrations with voltage will be slow, leading to a slope $\gamma<10 \mathrm{~V}^{-1}$ as observed. The main conclusion of our investigations up to now is that light and dark ideality factors considerably larger than 1 are explained only assuming SRH recombination (for instance via tail states), while a relatively constant and slow increase of the logarithm of carrier concentration with voltage with $\gamma<10 \mathrm{~V}^{-1}$ requires a considerable amount of trapped charge. Both features together automatically lead to a current scaling with average carrier concentration $n_{\mathrm{av}}^{\delta}$ with $\delta>2$. However, this should not be misinterpreted as a recombination rate according to Eq. (9), where $n$ and $p$ denote free carrier concentrations.

Figure 6 shows the CE experiments on the P3HT:PCBM cell in the dark plotted both as (a) carrier concentration versus voltage and as (b) current density versus carrier concentration. The tail recombination model fits relatively well in both cases. It should be noted that in this case one of the two plots is redundant since a good fit of the dark $J-V$ and either Figs. 6(a) or 6(b) automatically implies a good fit of the other part. With the same reasoning it becomes clear that a model with direct or Auger recombination can only reproduce one of the two depictions of the CE experiments well. Since it is impossible to reproduce the carrier density versus voltage plot in Fig. 6(a) and the current density versus carrier density plot in Fig. 6(b) at the same time, the fit for Auger and direct recombination was only done with the current density versus carrier density plot. Here, the fit is relatively accurate, with Auger recombination coming close to the expected slope. 

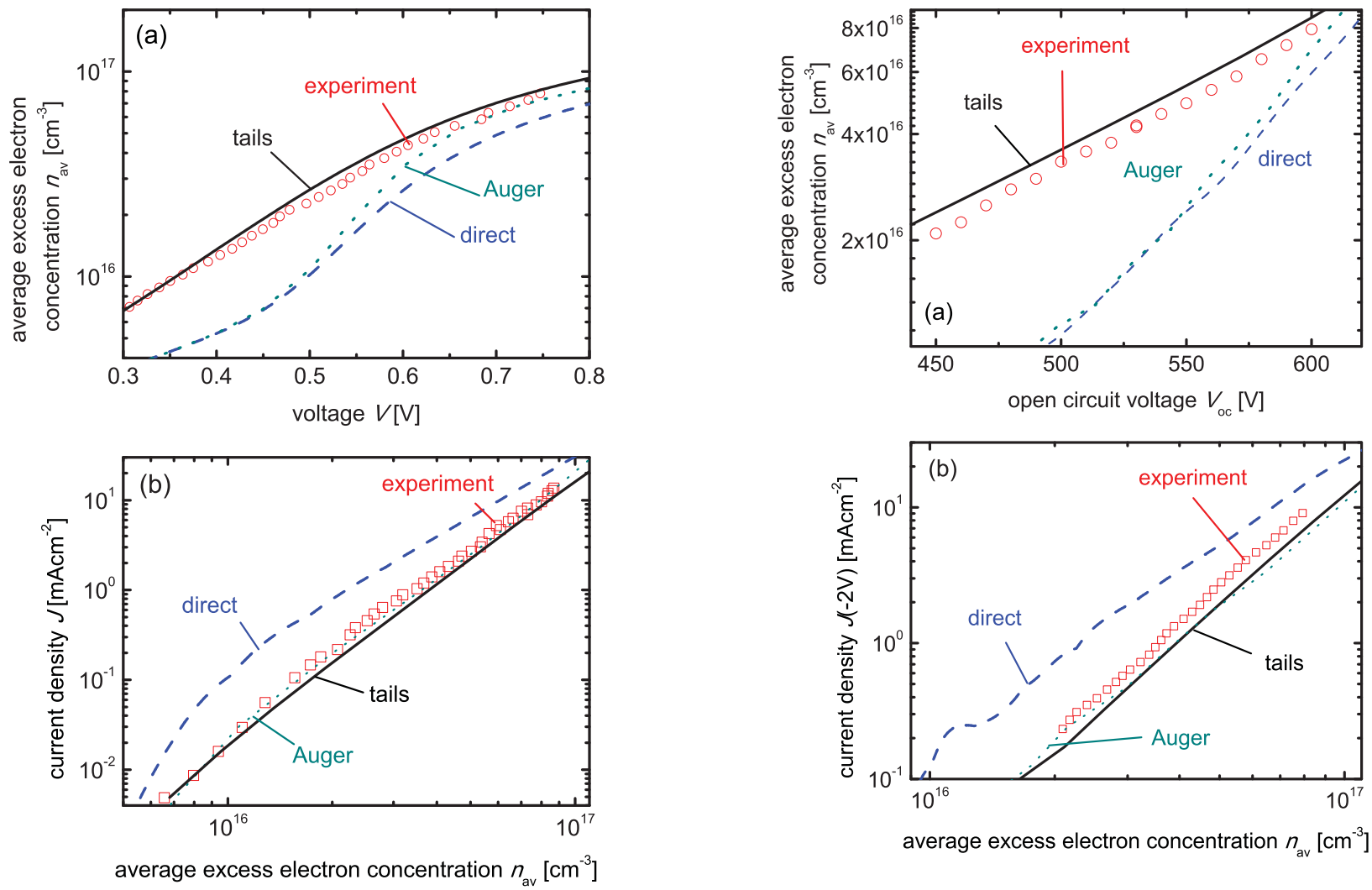

FIG. 6. (Color online) Comparison of experiment (symbols) and simulation for the CE measurements in the dark. For the simulations, again three recombination models have been used. Only the tail recombination model is capable of reproducing the (a) voltage dependence of the carrier concentration, while all models reproduce the (b) carrier dependence of the dark current density well.

Figure 7 shows a similar picture for the $\mathrm{CE}$ experiment under illumination. These experiments were not part of the fitting routine because this would have led to an enormous increase in computing time. Nevertheless, the simulations in case of the current density versus carrier density plot (b) fit relatively well in all cases, while the carrier density versus open-circuit voltage plot (a) shows strong deviations for direct and Auger recombination. Again, this is an expected outcome since Figs. 7(a) and 7(b) are connected by the intensity-dependent open-circuit voltage as shown in Fig. 4(c).

\section{ANALYTICAL APPROXIMATIONS AND POLARONIC EFFECTS}

Since this paper focuses on recombination at open-circuit conditions, where the carrier concentrations are reasonably homogenous, zero-dimensional simulations help to better understand the relation between the observable slopes when plotting current density, carrier concentration, and voltage against each other and the tail slope. If the tail slope is larger than $k T$, the concentration of trapped carriers scales approximately with ${ }^{69}$

$$
n_{\mathrm{t}} \propto n^{\frac{k T}{E_{\mathrm{UC}}}} .
$$

Thus, for the simplified case of a totally symmetric device, when both electron and hole concentration increase with half

of the voltage or quasi-Fermi level splitting, the concentration of trapped carriers scales with

$$
n_{\mathrm{t}} \propto \exp \left(\frac{q V}{2 E_{\mathrm{U}}}\right),
$$

where $E_{\mathrm{U}}=E_{\mathrm{UC}}=E_{\mathrm{UV}}$ is the tail slope of conduction- and valence-band tail. The parameter $\gamma$ is subsequently given by

$$
\gamma=1 /\left(2 E_{\mathrm{U}}\right) .
$$

Thus, typical values of $\gamma$ of slightly below 10 for P3HT:PCBM indicate tail slopes of approximately $E_{\mathrm{U}} \approx 2 k T$.

If we assume that recombination takes place between free electrons and trapped holes or the other way round, then recombination scales with

$$
\begin{aligned}
R & \propto n p_{\mathrm{t}} \propto \exp \left(\frac{q V}{2 k T}\right) \exp \left(\frac{q V}{2 E_{\mathrm{U}}}\right) \\
& =\exp \left(\frac{q V}{k T}\left[\frac{1}{2}+\frac{k T}{2 E_{\mathrm{U}}}\right]\right) .
\end{aligned}
$$

With the definition of the ideality factor, we arrive at ${ }^{63}$

$$
n_{\mathrm{id}}=\left(\frac{1}{2}+\frac{k T}{2 E_{\mathrm{U}}}\right)^{-1} \text {. }
$$


From the scaling of the recombination rate with carrier concentration,

$$
R \propto n p_{\mathrm{t}}=n^{E_{\mathrm{U}} / k T} n_{\mathrm{t}} p_{\mathrm{t}}=n_{\mathrm{t}}^{E_{\mathrm{U}} / k T+1},
$$

we determine the analytical approximation for the slope $\delta=$ $E_{\mathrm{U}} / k T+1$. Thus, it becomes clear that $\delta>2$ as observed in various experiments is easily explained by tail recombination with $E_{\mathrm{U}}>k T$. For recombination via any type of deep states (be it tail states or deep defects), we can state that, according to Eq. (16), slopes $\delta>2$ can be explained by tail slopes $E_{\mathrm{U}}>$ $k T n_{\text {id. }}$.

All the analytical relations detailed above are only valid for a perfectly symmetric device. If we calculate the tail slope from the measured values of $\gamma$ and $n_{\text {id }}$ using Eqs. (19) and (21), we obtain different values, namely, $\sim 50 \mathrm{meV}$ from $\gamma=9.3 \mathrm{~V}^{-1}$ and $\sim 100 \mathrm{meV}$ from $n_{\mathrm{id}}=1$.6. One possible explanation for this discrepancy is that the tail slopes are strongly asymmetric implying that the analytical relations do not hold anymore. This explanation has been used for the fits (cf. Table I), but it does not have to be the correct explanation. Other possible explanations might be (i) a more complicated density of states with additional deeper states that affect recombination and increase the ideality factor but that are too deep to be extracted during the $\mathrm{CE}$ experiment, or (ii) polaronic effects.

To analyze the impact of polaronic effects on the slopes $\gamma$ and $n_{\text {id }}$, we combined the idea of the SRH statistics with a rate equation according to Marcus theory. The processes at the basis of SRH statistics are CT reactions. Consider the electron trapping process labeled $\beta_{\mathrm{n}}^{0}$ in Fig. 2. In the traditional application of SRH statistics, this represents the capture of a free electron by an immobile trap state. In this context the capture rate coefficient is related to the thermal velocity of the free charge carrier. In the context of organic semiconductors, where charges are significantly more localized, it is more helpful to imagine this process as the transfer of an electron from a site with high energy to a site with a lower energy. This sort of CT reaction is often described using rate equations from Marcus theory. ${ }^{70}$ This does not fundamentally affect the relevance of SRH statistics, but it does change the rates used to describe electron capture and electron emission. In particular, in traditional SRH the downhill processes (electron capture and hole emission) are independent of the energy difference between the trap level and the respective band edge, whereas the uphill processes (electron emission and hole capture) are decelerated by the factor $\exp (-\Delta E / k T)$ to comply with a detailed balance, where $\Delta E$ is the energetic difference between the trap and the respective band edge. The rates in classical SRH theory are thus basically Miller-Abrahams hopping rates. In Marcus theory uphill and downhill processes are described by the same equation,

$$
\beta=\frac{\beta_{0}}{\sqrt{\lambda k T}} \exp \left(-\frac{[\Delta E-\lambda]^{2}}{4 \lambda k T}\right),
$$

where $\beta_{0}$ is a prefactor depending on the wave-function overlap of the two states and $\lambda$ is the reorganization energy. Note that Eq. (23) is detailed balance compatible as well, because it can be written as

$$
\beta=\beta_{00}\left(\lambda, \Delta E^{2}\right) \exp \left(\frac{-\Delta E}{2 k T}\right),
$$

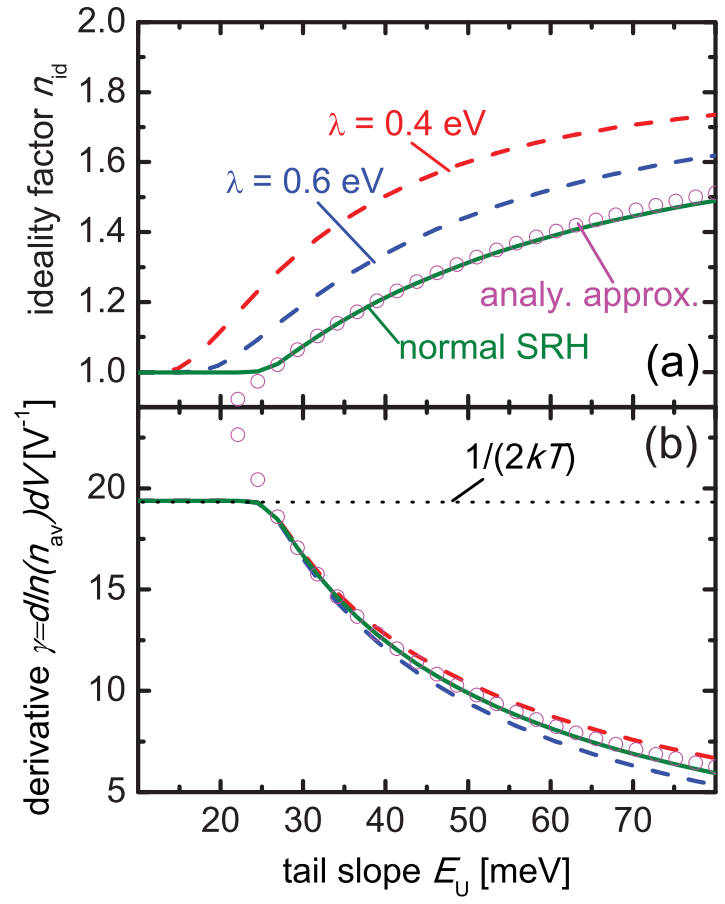

FIG. 8. (Color online) (a) Ideality factor $n_{\mathrm{id}}$ and (b) slope $\gamma=d \ln \left(n_{\text {av }}\right) / d V$ as a function of tail slope $E_{\mathrm{U}}$ (same for the conduction- and valence-band tail) for normal SRH statistics (solid line), for Marcus-type hopping rates combined with SRH statistics (dashed lines, for two different reorganization energies $\lambda$ ), and for the analytical approximations Eqs. (19) and (21) (open circles). While the ideality factor is considerably larger at a given tail slope for the Marcus-type hopping rates than for the normal SRH statistics, the slope $\gamma$ is roughly the same. Note that the analytical approximations are only valid for $E_{\mathrm{U}}>k T$ and give unreasonable values for $E_{\mathrm{U}}<k T$.

i.e., the ratio of uphill to downhill rates is proportional to $\exp (-\Delta E / k T)$ as for the normal SRH statistics.

Figure 8 compares the normal SRH statistics with the one with Marcus-type rates and the analytical approximations [Eqs. (19) and (21)]. The simulations were done in zero dimensions using symmetric parameters for electrons and holes. Here transport is not relevant, but the zero-dimensional simulation with normal SRH statistics fit very well to one-dimensional drift diffusion simulations at open circuit. Figure 8(a) compares the ideality factor as a function of tail slope for conduction- and valence-band tails. While the simulation with the normal SRH statistics fits very well to the analytical approximation of van Berkel et al. ${ }^{63}$ [Eq. (21)], the simulation with the combination of Marcus theory and SRH statistics produces higher ideality factors. In addition, the lower the reorganization energy $\lambda$, the higher are the ideality factors.

Figure 8(b) shows that the voltage dependence of the carrier concentration is hardly affected by the choice of the rates or the value of the reorganization energy. For typical tail slopes of $50 \mathrm{meV}$ needed to explain the voltage dependence of carrier concentration $(\gamma)$, the experimentally observed ideality factor of 1.6 is reached for reorganization energies $\sim 0.4 \mathrm{eV}$.

Thus, there are several possible explanations for the experimental observations that all share some common features: 
(i) There have to be states below the interfacial band gap which affect transport and recombination. (ii) SRH statistics can be used to calculate the recombination rates, which implies that recombination takes place between a more mobile carrier and trapped carriers. However, current-voltage curves and CE measurements alone are not sufficient to exactly determine the density of states. Thus, other methods for a more detailed determination of the subgap density of states are necessary.

\section{ALTERNATIVE INTERPRETATIONS OF $\boldsymbol{n}_{\text {id }}>1$}

The reasoning of this article is based heavily on the explanation of the dark and light ideality factor of the P3HT:PCBM solar cell under investigation with SRH recombination. This conclusion could be questioned if there are alternative, plausible explanations for the larger-than-1 ideality factor. One explanation that has been suggested for higher ideality factors ${ }^{71,72}$ in disordered semiconductors is the Einstein relation,

$$
D_{\mathrm{n}, \mathrm{p}}=\mu_{\mathrm{n}, \mathrm{p}} k T / q,
$$

which does not hold anymore, when the Fermi energy $E_{f}$ comes close to the peak of the energy-dependent charge concentration. In the case of a Gaussian density of states or exponential band tails, a large amount of charge may be well below the peak of the Gaussian density of states or below the band gap in the case of the exponential tails. In these cases Eq. (25) has to be replaced by a generalized Einstein relation that reads (e.g., for electrons) ${ }^{73}$

$$
D_{\mathrm{n}}=\mu_{\mathrm{n}} \frac{n}{q \frac{d n}{d E_{\mathrm{f}}}}=\mu_{\mathrm{n}} \frac{1}{q \frac{d \ln n}{d E_{\mathrm{f}}}} .
$$

Reference 71 describes the calculation of $d \ln n / d E_{\mathrm{f}}$ or $d \ln n / d V$ for a Gaussian density of states and for exponential bands. As in the case of SRH recombination, the ideality factor increases above unity in case the electron concentration does not increase as in a perfect (not disordered or degenerate) crystal with $n \propto \exp (q V / k T)$ (for $n \ll p$ ) or $n \propto \exp (q V / 2 k T)$ for $n=p$. So as illustrated in more detail in Appendix B, both SRH recombination and the approach described in Ref. 71 result in ideality factors above 1, since in both cases the concentration of either electrons or holes scales with voltage in a different way as a nondegenerate carrier population in a band would do. The differences of both models are then rather minor and deal with the question of how to treat the thermalization of carriers below the band gap. While Ref. 71 assumes the same Fermi level for all states, the SRH theory includes the calculation for the occupation of tail states, which leads to an occupation function having a mathematical form that is similar to a Fermi-Dirac distribution with two quasi-Fermi levels for electrons in the conduction-band tail and two Fermi levels for holes in the valence-band tail. ${ }^{74}$

\section{LIMITATIONS OF THE MODEL AND DIRECTIONS FOR FURTHER WORK}

Since all simulations were carried out with a commercial solar-cell simulator (ASA), the model is restricted to the options available in this software. Thus, there are a number of effects that are not taken into account and simplifications that have to be made. The shape of the density of states for the trapped charge is assumed to decay exponentially into the band gap. Drift-diffusion simulations with Gaussian band tails cannot be made with ASA.

In addition, the model is completely one dimensional and does not explicitly take the morphology of the device into account. A particular problem is that trapping of carriers may happen everywhere in the device, while recombination only takes place at the donor-acceptor heterointerfaces. This can be taken into account by choosing different capture rate coefficients for trapping and for recombination.

The model distinguishes between free and trapped carriers, where the free ones have a constant band mobility and the trapped carriers are completely immobile. Thus, the effective mobility depends implicitly on the ratio of free to trapped carriers. The assumption of an abrupt mobility edge is certainly wrong for any disordered solar cell. However, in the case of amorphous silicon it does not affect the result since the transport of the mobile carriers is much more efficient than the transport of the carriers close to the Fermi level. For organic solar cells this difference might not be large enough, and the effect of a nonabrupt mobility edge might have to be included in future work.

Although the model can reproduce ideality factors $1<$ $n_{\text {id }}<2$, any larger ideality factors that are sometimes observed cannot be explained directly. One possible explanation of this behavior is the recombination of two carriers that are both trapped. In this case the concentrations of both partners involved in recombination have a voltage dependence that is not described by Boltzmann approximations. Such tail-to-tail or tail-to-defect recombination is not taken into account in ASA, assuming that recombination involving one free carrier should be dominant because the free carrier will more easily find a trapped one for recombination. For $p-i$ - $n$ type solar cells with ideality factors $n_{\text {id }}>2$ this assumption is possibly wrong, which means that tail-to-tail recombination should be included in drift-diffusion models.

Another shortcoming of the present interpretation of the CE data is the assumption that all charge in the tails is collected. To investigate this assumption further, it would be necessary to develop experimental methods to determine the energy-dependent lifetime of the carriers in the tails in order to calculate a demarcation energy that separates collected from uncollected carriers.

\section{CONCLUSIONS}

The disordered nature of organic solar cells requires a more complex approach to model recombination in these devices than would be necessary for crystalline semiconductors. Here, we find that a recombination model typically used for thinfilm silicon solar cells is sufficient to reproduce the voltage dependence of carrier concentration and recombination current in a P3HT:PCBM solar cell. The main feature of the model is the inclusion of SRH recombination via a distribution of states in the band gap. Effectively, the recombination no longer takes place between two free carriers whose concentrations are determined by Boltzmann approximations, but instead, the recombination takes place between free electrons and trapped holes or vice versa. The concentration of trapped electrons or 
holes is then no longer determined by Boltzmann statistics but instead by SRH statistics, and has a distinctly different voltage dependence than expected for free carriers. This voltage dependence of carrier concentration is the key to explain both the charge extraction measurements and the observed ideality factors above unity. In particular, the inclusion of disorder provides a simple explanation why recombination increases with more than the square of the carrier concentration while the ideality factor is above unity at the same time. These two results are contradictory in the absence of disorder. Future work needs to address the detailed experimental determination of the unknown parameters describing trapping and recombination in subband-gap states and especially of the density of states below the interfacial band gap.

\section{ACKNOWLEDGMENTS}

The authors would like to thank K. Taretto and M. Soldera (Uni. Neuquen) for discussion and inspiration in the early stage of the work and C. Shuttle (UCSB) and J. Durrant (Imperial College) for sharing the experimental data. In addition, we want to thank R. Mackenzie, G. Dibb, A. Maurano, and D. Credgington (Imperial College) for fruitful discussions on modeling and charge extraction. T.K. acknowledges partial support by an Imperial College Junior Research Fellowship. J. N. acknowledges the support of the UK Engineering and Physical Sciences Research Council through the Supergen (EP/031088/1) and Nanotechnology Grand Challenges (EP/F056710/1, EP/F056389/1) programmes.

\section{APPENDIX A: IDEALITY FACTOR IN CASE OF DIRECT AND AUGER RECOMBINATION}

In the following, we will show analytically why the ideality factor for direct recombination is always 1 and for Auger recombination $2 / 3<n_{\mathrm{id}}<1$. The ideality factor is always determined from the dark $J-V$ curve in the voltage range where low mobility and series resistance effects have no effect on the dark $J-V$ curve, or from the illumination-dependent open-circuit voltage. In the latter case it is not important whether mobilities and external series resistances are high or low, since there is no current flowing (except for the surface recombination current ${ }^{75-77}$ ). Thus, in order to derive the ideality factor, we can assume that the quasi-Fermi levels are flat, because we restrict ourselves to low voltages or opencircuit situations. Direct recombination is the recombination of free electrons and free holes in the definition of "free" used in the paper, which is that the concentration of free electrons and holes is calculated with the Boltzmann approximation, meaning that $n p \sim \exp (q V / k T)$. For direct recombination, the recombination current density is then

$$
J=q \int_{0}^{d} k\left(n p-n_{\mathrm{i}}^{2}\right) d x=q k n_{\mathrm{i}}^{2} d\left[\exp \left(\frac{q V}{k T}\right)-1\right] .
$$

Thus, the ideality factor is 1 , i.e., $J$ scales with $\exp (q V / k T)$, because both the electron and hole concentration scale with voltage according to Boltzmann statistics.

In the case of Auger recombination, we distinguish between the case where one of the carrier concentrations is much larger than the other, and the case where they are roughly identical. For $n \gg p$, e.g., the recombination current density is

$J=q \int_{0}^{d} C\left(n^{2} p+p^{2} n\right) d x \approx q C n_{\mathrm{i}}^{2} n d\left[\exp \left(\frac{q V}{k T}\right)-1\right]$.

Usually, the voltage dependence of the majority-carrier concentration (here $n$ ) is small, so the ideality factor will be $n_{\text {id }} \leqslant 1$. For the case $n \approx p$, the Auger recombination current density is

$$
J=q \int_{0}^{d} 2 C n^{3} d x \approx q 2 C n_{\mathrm{i}}^{2} d\left[\exp \left(\frac{3 q V}{2 k T}\right)-1\right],
$$

leading to an ideality factor $n_{\mathrm{id}}=2 / 3$. The case described by Eq. (A2) corresponds to a typical dark $J-V$ curve, while the latter case better explains the behavior under open-circuit conditions.

\section{APPENDIX B: IDEALITY FACTOR FOR SRH RECOMBINATION EXPLAINED AS DUE TO THE DIFFERENT VOLTAGE DEPENDENCE OF TRAPPED CARRIER CONCENTRATIONS AS OPPOSED TO FREE CARRIER CONCENTRATIONS}

To illustrate the fact that the ideality factor for $\mathrm{SRH}$ recombination is solely due to the voltage dependence of carrier concentrations involved in recombination, let us consider the simple example of a semiconductor with free carrier concentrations $n$ and $p$ that follow Boltzmann statistics. The semiconductor has a single trap sufficiently deep in the band gap that emission processes can be neglected. Then the electron recombination rate is

$$
R=\beta_{\mathrm{n}} n N_{\mathrm{t}}(1-f),
$$

where $1-f$ is the probability that the defect with concentration $N_{\mathrm{t}}$ is occupied by a hole and $\beta_{\mathrm{n}}$ is the capture rate coefficient for electrons. So the concentration $p_{\mathrm{t}}$ of holes on the defect level would be $p_{\mathrm{t}}=N_{\mathrm{t}}(1-f)$. Thus, the SRH recombination rate $R=\beta_{\mathrm{n}} n p_{\mathrm{t}}$ looks practically the same as a direct recombination rate, with the only difference being that the hole concentration $p_{\mathrm{t}}$ involved in the recombination process is not a concentration of holes in a band where Boltzmann approximations are valid, but instead it is the concentration of holes in a localized state. This concentration depends on the concentration in the bands via $1-f=p \beta_{\mathrm{p}} /\left(n \beta_{\mathrm{n}}+p \beta_{\mathrm{p}}\right){ }^{78}$ Thus, we obtain the typical SRH recombination rate,

$$
R=\beta_{\mathrm{n}} \beta_{\mathrm{p}} N_{\mathrm{t}} \frac{n p}{n \beta_{\mathrm{n}}+p \beta_{\mathrm{p}}} .
$$

For $n \approx p$ as in the middle of $p-i-n$ junction solar cells or in the space-charge region ${ }^{79}$ of $p-n$ junction solar cells, the recombination rate $R \propto n \propto \exp (q V / 2 k T)$, leading to the typical ideality factor of 2 . Here we assumed that $n p \propto$ $\exp (q V / k T)$, i.e., that the Boltzmann approximation and the effective density of states approach are both appropriate for the carriers in the bands. The ideality factor of 2 arises only from the fact that the hole concentration on the defect does not scale with voltage the same way as the carriers in the bands do. 


\section{APPENDIX C: MONOMOLECULAR VERSUS BIMOLECULAR RECOMBINATION}

Recent publications ${ }^{55,80}$ on recombination in organic bulk heterojunction solar cells discussed the question whether the dominant recombination mechanism is bimolecular or monomolecular. If monomolecular and bimolecular are defined in terms of how the recombination rate scales with carrier concentration (monomolecular: $R \propto n^{1}$; bimolecular: $R \propto n^{2}$ ), then results from CE hint at recombination rates $R \propto n^{\delta}$ with $\delta>2$, i.e., bimolecular or higher order. In contrast, ideality factors $n_{\text {id }}>1$ on first sight hint at a bimolecular or lower order. An ideality factor $n_{\text {id }}=2$, e.g., is (in the Boltzmann approximation) consistent with $R \propto$ $\sqrt{n p} \propto \exp (q V / 2 k T)$, i.e., monomolecular recombination. This apparent discrepancy is resolved when you take into account that the carrier concentration scales with voltage not according to the Boltzmann approximation but much slower depending on the slope of the tail states. Then it is possible that the recombination rate $R \propto \exp (q V / 2 k T)$ and at the same time $R \propto n^{\delta}$ holds with $\delta>2$. The question, whether such recombination via tail states is monomolecular or bimolecular, is then purely a matter of definition of these two terms. Thus, any future discussion on nongeminate recombination using the terms bimolecular and monomolecular will benefit from a proper definition of these terms that takes both the proportionality of recombination rate and measured charge density as well as the ideality factor into account.

Note that in some publications ${ }^{46,81,82}$ the terms bimolecular and monomolecular are defined in a mechanistic sense and do not define a rate order. In this case, bimolecular means that an electron on one molecule recombines with a hole on another molecule, which makes bimolecular recombination a synonym for nongeminate recombination. Monomolecular is then used as a synonym for geminate recombination. Within this terminology, all recombination discussed in this paper is bimolecular.
${ }^{1}$ R. Gaudiana and C. J. Brabec, Nat. Photonics 2, 287 (2008).

${ }^{2}$ C. J. Brabec and J. R. Durrant, MRS Bull. 33, 670 (2008).

${ }^{3}$ V. Shrotriya, Nat. Photonics 3, 447 (2009).

${ }^{4}$ M. Pagliaro, R. Ciriminna, and G. Palmisano, ChemSusChem. 1, 880 (2008).

${ }^{5}$ T. Söderström, F.-J. Haug, V. Terrazoni-Daudrix, and C. Ballif, J. Appl. Phys. 107, 014507 (2010).

${ }^{6}$ M. B. Schubert and J. H. Werner, Mater. Today 9, 42 (2006).

${ }^{7}$ N. S. Sariciftci, L. Smilowitz, A. J. Heeger, and F. Wudl, Science 258, 1474 (1992).

${ }^{8}$ G. Yu, J. Gao, J. C. Hummelen, F. Wudl, and A. J. Heeger, Science 270, 1789 (1995).

${ }^{9}$ B. A. Gregg and M. C. Hanna, J. Appl. Phys. 93, 3605 (2003).

${ }^{10}$ B. A. Gregg, J. Phys. Chem. B 107, 4688 (2003).

${ }^{11}$ T. Kirchartz, J. Mattheis, and U. Rau, Phys. Rev. B 78, 235320 (2008).

${ }^{12}$ S. H. Park, A. Roy, S. Beaupré, S. Cho, N. Coates, J. S. Moon, D. Moses, M. Leclerc, K. Lee, and A. J. Heeger, Nat. Photonics 3, 297 (2009).

${ }^{13}$ K. Maturová, S. S. van Bavel, M. M. Wienk, R. A. J. Janssen, and M. Kemerink, Nano Lett. 9, 3032 (2009).

${ }^{14}$ K. Vandewal, K. Tvingstedt, A. Gadisa, O. Inganäs, and J. V. Manca, Nat. Mater. 8, 904 (2009).

${ }^{15}$ T. Kirchartz, K. Taretto, and U. Rau, J. Phys. Chem. C 113, 17958 (2009).

${ }^{16}$ C. G. Shuttle, A. Maurano, R. Hamilton, B. O'Regan, J. C. de Mello, and J. R. Durrant, Appl. Phys. Lett. 93, 183501 (2008).

${ }^{17}$ A. Sanchez-Diaz, M. Izquierdo, S. Filippone, N. Martin, and E. Palomares, Adv. Funct. Mater. 20, 2695 (2010).

${ }^{18}$ J. C. Blakesley, H. S. Clubb, and N. C. Greenham, Phys. Rev. B 81, 045210 (2010).

${ }^{19}$ J. A. Anta, Energy Environ. Sci. 2, 387 (2009).

${ }^{20}$ C. G. Shuttle, R. Hamilton, J. Nelson, B. C. O'Regan, and J. R. Durrant, Adv. Funct. Mater. 20, 698 (2010).

${ }^{21}$ J. Nelson, Phys. Rev. B 67, 155209 (2003).

${ }^{22}$ M. P. Eng, P. F. Barnes, and J. R. Durrant, J. Phys. Chem. Lett. 1, 3096 (2010).
${ }^{23}$ M. M. Mandoc, F. B. Kooistra, J. C. Hummelen, B. de Boer, and P. W. M. Blom, Appl. Phys. Lett. 91, 263505 (2007).

${ }^{24}$ G. Garcia-Belmonte and J. Bisquert, Appl. Phys. Lett. 96, 113301 (2010).

${ }^{25}$ M. Tachiya and K. Seki, Phys. Rev. B 82, 085201 (2010).

${ }^{26}$ N. C. Giebink, G. P. Wiederrecht, M. R. Wasielewski, and S. R. Forrest, Phys. Rev. B 82, 155305 (2010).

${ }^{27}$ N. C. Giebink, B. E. Lassiter, G. P. Wiederrecht, M. R. Wasielewski, and S. R. Forrest, Phys. Rev. B 82, 155306 (2010).

${ }^{28}$ V. D. Mihailetchi, L. J. A. Koster, J. C. Hummelen, and P. W. M. Blom, Phys. Rev. Lett. 93, 216601 (2004).

${ }^{29}$ D. W. Sievers, V. Shrotriya, and Y. Yang, J. Appl. Phys. 100, 114509 (2006).

${ }^{30}$ H. H. P. Gommans, M. Kemerink, J. M. Kramer, and R. A. J. Janssen, Appl. Phys. Lett. 87, 122104 (2005).

${ }^{31}$ L. J. A. Koster, V. D. Mihailetchi, R. Ramaker, and P. W. M. Blom, Appl. Phys. Lett. 86, 123509 (2005).

${ }^{32}$ L. J. A. Koster, V. D. Mihailetchi, and P. W. M. Blom, Appl. Phys. Lett. 88, 093511 (2006).

${ }^{33}$ J. D. Kotlarski, P. W. M. Blom, L. J. A. Koster, M. Lenes, and L. H. Slooff, J. Appl. Phys. 103, 084502 (2008).

${ }^{34}$ C. Deibel, A. Wagenpfahl, and V. Dyakonov, Phys. Rev. B 80, 075203 (2009).

${ }^{35}$ A. Wagenpfahl, D. Rauh, M. Binder, C. Deibel, and V. Dyakonov, Phys. Rev. B 82, 115306 (2010).

${ }^{36}$ C. L. Braun, J. Chem. Phys. 80, 4157 (1984).

${ }^{37}$ M. Wojcik and M. Tachiya, J. Chem. Phys. 130, 104107 (2009).

${ }^{38}$ M. Hilzcer and M. Tachiya, J. Phys. Chem. C 114, 6808 (2010).

${ }^{39}$ L. J. A. Koster, E. C. P. Smits, V. D. Mihailetchi, and P. W. M. Blom, Phys. Rev. B 72, 085205 (2005).

${ }^{40}$ C. Deibel, A. Wagenpfahl, and V. Dyakonov, Phys. Status Solidi RRL 2, 175 (2008).

${ }^{41}$ R. A. Street, S. Cowan, and A. J. Heeger, Phys. Rev. B 82, 121301(R) (2010).

${ }^{42}$ C. G. Shuttle, R. Hamilton, B. C. O'Regan, J. Nelson, and J. R. Durrant, Proc. Natl. Acad. Sci. 107, 16448 (2010). 
${ }^{43}$ F. C. Jamieson, T. Agostinelli, H. Azimi, J. Nelson, and J. R. Durrant. J. Phys. Chem. Lett. 1, 3306 (2010).

${ }^{44}$ C. Deibel, T. Strobel, and V. Dyakonov, Phys. Rev. Lett. 103, 036402 (2009).

${ }^{45}$ C. G. Shuttle, B. O'Regan, A. M. Ballantyne, J. Nelson, D. D. C. Bradley, J. de Mello, and J. R. Durrant, Appl. Phys. Lett. 92, 093311 (2008).

${ }^{46}$ C. G. Shuttle, B. O'Regan, A. M. Ballantyne, J. Nelson, D. D. C. Bradley, and J. R. Durrant, Phys. Rev. B 78, 113201 (2008).

${ }^{47}$ G. Juška, K. Genevičius, G. Sliaužys, N. Nekrašas, and R. Österbacka, J. Non-Cryst. Solids 354, 2858 (2008).

${ }^{48}$ G. Juška, K. Genevičius, N. Nekrašas, G. Sliaužys, and G. Dennler, Appl. Phys. Lett. 93, 143303 (2008).

${ }^{49}$ C. Deibel, A. Baumann, and V. Dyakonov, Appl. Phys. Lett. 93, 163303 (2008).

${ }^{50}$ G. Juška, K. Genevičius, N. Nekrašas, G. Sliaužys, and R. Österbacka, Appl. Phys. Lett. 95, 013303 (2009).

${ }^{51}$ R. E. I. Schropp and M. Zeman, Amorphous and Microcrystalline Silicon Solar Cells: Modeling, Materials and Device Technology (Kluwer, Norwell, 1998), p. 117.

${ }^{52}$ R. A. Street, J. E. Northrup, and A. Salleo, Phys. Rev. B 71, 165202 (2005).

${ }^{53}$ G. Garcia-Belmonte, P. P. Boix, J. Bisquert, M. Sessolo, and H. J. Bolink, Sol. Energy Mater. Sol. Cells 94, 366 (2010).

${ }^{54}$ G. Garcia-Belmonte, P. P. Boix, J. Bisquert, M. Lenes, H. J. Bolink, A. La Rosa, S. Filippone, and N. Martin, J. Phys. Chem. Lett. 1, 2566 (2010).

${ }^{55}$ R. A. Street, M. Schoendorf, A. Roy, and J. H. Lee, Phys. Rev. B 81, 205307 (2010).

${ }^{56}$ J. A. Willemen, Dissertation, TU Delft, 1998, p. 100 [http://repository.tudelft.nl/assets/uuid:0771d543-af5f-4579-8a350b68d34a1334/emc_willemen_19981016.PDF].

${ }^{57}$ R. N. Hall, Phys. Rev. 87, 387 (1952).

${ }^{58}$ W. Shockley and W. T. Read, Phys. Rev. 87, 835 (1952).

${ }^{59}$ For a derivation of Eq. (10) see, for instance, J. A. Willemen, Dissertation, TU Delft, 1998, p. 45 [http://repository. tudelft.nl/assets/uuid:0771d543-af5f-4579-8a35-0b68d34a1334/ emc_willemen_19981016.PDF]; B. E. Pieters TU Delft, Dissertation, 2008, p. 30 [http://repository.tudelft.nl/assets/uuid: 83bc5ff4-8e33-4962-9014-68cfbad8226f/pieters_20081111.pdf].

${ }^{60}$ L. Jiang, J. H. Lyou, S. Rane, E. A. Schiff, Q. Wang, and Q. Yuan, Mater. Res. Soc. Symp. Proc. 609, A18.3.1-12 (2000).
${ }^{61}$ K. Vandewal, K. Tvingstedt, A. Gadisa, O. Inganäs, and J. V. Manca, Phys. Rev. B 81, 125204 (2010).

${ }^{62}$ B. E. Pieters, T. Kirchartz, T. Merdzhanova, and R. Carius, Sol. Ener. Mater. Sol. Cells 94, 1851 (2010).

${ }^{63}$ C. van Berkel, M. J. Powell, A. R. Franklin, and I. D. French, J. Appl. Phys. 73, 5264 (1993).

${ }^{64}$ T. Walter, R. Herberholz, and H. W. Schock, Solid State Phenom. 51-52, 309 (1996).

${ }^{65}$ Further details on this device are available in C. G. Shuttle, A. Maurano, R. Hamilton, B. O'Regan, J. C. de Mello, and J. R. Durrant, Appl. Phys. Lett. 93, 183501 (2008).

${ }^{66}$ B. E. Pieters, J. Krc, and M. Zeman, Conference Record of 2006 IEEE World Photovoltaics, p. 1513 (unpublished).

${ }^{67}$ MATLAB function fminsearch.

${ }^{68}$ J. A. Nelder and R. Mead, Comput. J. 7, 308 (1965).

${ }^{69}$ G. J. Adriaenssens, S. D. Baranovskii, W. Fuhs, J. Jansen, and Ö. Öktü, Phys. Rev. B 51, 9661 (1995).

${ }^{70}$ V. Coropceanu, J. Cornil, D. A. da Silva Filho, Y. Olivier, R. Silbey, and J. L. Brédas, Chem. Rev. 107, 926 (2007).

${ }^{71}$ N. Tessler and Y. Roichman, Organic Electronics 6, 200 (2005).

${ }^{72}$ K. Harada, A. G. Werner, M. Pfeiffer, C. J. Bloom, C. M. Elliott, and K. Leo, Phys. Rev. Lett. 94, 036601 (2005).

${ }^{73}$ Y. Roichman and N. Tessler, Appl. Phys. Lett. 80, 1948 (2002).

${ }^{74}$ J. G. Simmons and G. W. Taylor, Phys. Rev. B 4, 502 (1971).

${ }^{75}$ T. Kirchartz, B. E. Pieters, K. Taretto, and U. Rau, J. Appl. Phys. 104, 094513 (2008).

${ }^{76}$ A. Wagenpfahl, C. Deibel, and V. Dyakonov, IEEE J. Sel. Top. Quantum Electron. 16, 1759 (2010).

${ }^{77}$ T. Kirchartz, B. E. Pieters, K. Taretto, and U. Rau, Phys. Rev. B 80, 035334 (2009).

${ }^{78}$ Cf. B. E. Pieters, Dissertation, TU Delft, 2008, p. 31, Eq. (3.31), with $e_{n}=e_{p}=0$.

${ }^{79}$ For a more detailed discussion, see, e.g., C. T. Sah, R. N. Noyce, and W. Shockley, Proc. Inst. Radio Eng. 45, 1228 (1957).

${ }^{80}$ C. Deibel and A. Wagenpfahl, Phys. Rev. B 82, 207301 (2010).

${ }^{81}$ A. Maurano, R. Hamilton, C. G. Shuttle, A. M. Ballantyne, J. Nelson, B. O'Regan, W. Zhang, I. McCulloch, H. Azimi, M. Morana, C. J. Brabec, and J. R. Durrant, Adv. Mater. 22, 4987 (2010).

${ }^{82}$ T. M. Clarke and J. R. Durrant, Chem. Rev. 110, 6736 (2010). 